\title{
Massive Regional Texture Extraction for Aerial and Natural Images
}

\author{
S. Rizwana, Dr. S. Pannirselvam \\ Research Scholar, Manonmaniam Sundaranar University, Tirunelveli. \\ Associate Professor \& HOD, Erode Arts and Science College, Erode.
}

\begin{abstract}
In recent years, image processing acts as an objective for the evaluation of images. Texture is a primary feature which presents considerable information for image classification, and is an imperative content utilized in content-based image retrieval (CBIR) system. To employ texture-based image database retrieval, texture segmentation strategies are required to segment textured regions from arbitrary images in the database. Texture segmentation has been accepted as a complex crisis in image analysis. The main objective of image segmentation is cluster pixeling the regions equivalent to individual surfaces, objects, or ordinary parts of objects and to simplify and/or change the representation of an image into something that is more meaningful and easier to analyze.

To enhance the texture image segmentation, in this work, we are going to implement a multitude regional texture extraction process for image segmentation. At first, natural and aerial images are extracted from the dataset and present region based segmentation process. Then a multitude regional texture extraction is proposed by implementing a local threshold values. An extraction of regions are accomplished by the respective dimensions. The algorithm provides a less natural metrics awareness in a minimum user interaction environment. The shape and size of the growing regions depend on look up table entries. The experimental evaluation is conducted with training samples of natural and aerial images to show the performance of multitude textural extraction for more efficient image segmentation with sharp demarcation of edge portions along with intensity levels.
\end{abstract}

Key Words: Image Segmentation, Texture Extraction, Region Growing, Natural and Aerial Images

\section{Introduction}

Huge image collections are accessible at the moment in different areas such as digital propagation, distraction, learning, and multimedia communication. With this enormous quantity of image, more proficient storage, indexing and recovery of visual information are robustly vital. The customary strategy for probing digital image databases needs a substantial stage of human effort, and is habitually subjective. These troubles guides to content based image retrieval (CBIR) scheme which recovers images on the base of routinely consequent features such as color, texture and shape. CBIR not only eradicates physical processing for image indexing but also gives habitual indexing consistent with image contents.

Among contents based features, texture is an essential feature which presents considerable information for image categorization. Although no specific description so far, textures regularly submit to homogeneous patterns or spatial preparations of pixels that regional intensity or color only does not adequately explain. Texture explains the content of numerous real world images, for instance clouds, bricks, etc.

An image in the database can be measured as mosaics of textured regions, and features of every textured section can be utilized to guide the entire database for recovery purpose. To realize such texture-based image reclamation, the first job is to fragment textured regions from subjective images.

Texture segmentation acts as an imperative role in both image analysis and computer vision. It comprises of dividing the input image into associated regions which are consistent with regard to a texture property. Texture segmentation has been renowned as a complex problem and has been endeavored in abundant ways. Generally, these techniques can be categorized into

- Feature-based methods,

- Model-based methods, and

- Structure-based methods.

Structure-based methods split the images under the statement that the textures in the image have measurable prehistoric elements, approved consistent with assignment rules. In feature-based methods, regions with fairly constant texture uniqueness are required.

Model-based techniques assume primary processes for textures and segments employing definite constraints of these processes. Model based techniques can be measured as a subclass of feature-based systems because model factors are employed as texture features. From a different point of view, texture segmentation can be categorized into supervised and unsupervised texture segmentation. Supervised texture segmentation is 
controlled to a collection of identified textures. Unsupervised texture segmentation is a difficult task since the lack of previous knowledge concerning the texture in the image is positioned.

Along with the segmentation techniques, texture segmentation can be classified into two classes:

$\circ$ Region-based and

○ Boundary based.

Boundary-based technique seeks to notice the dissimilarities of texture in neighboring regions whilst region-based strategy tries to recognize regions with consistent texture.

The major advantage of Region growing over conventional segmentation is the borders of regions found by region growing are perfectly thin and connected. And also the algorithm is very stable with respect to noise. The important thing is that membership in a region can be based on multiple criteria. We can take advantage of several image properties, such as low gradient or gray level intensity value, at once.

\section{Literature Review}

In image segmentation, a simple method is implemented termed as thresholding. The simple technique is processed based on a clip-level (or a threshold value) to convert a gray-scale image into a binary image.The main obejctive of this simple image segmentation method is to choose the threshold value when multiple-levels of the portions are selected.

Normally, in image processing, a well-developed technique called Edge detection is used. The edge detection process has a sharp adjustment in intensity of region boundaries which are closely related. Edge detection methods are the abse for all segmentation technique. The edges identified by edge detection are often disconnected. Closed region boundaries are necessary to segment an object from an image.

Segmentation [2] is usually a process of partitioning a digital image into multiple segments the major objective of segmentation is to simplify and/or change the representation of an image into something that is more meaningful and easier to analyze the medical images [3]. Image segmentation is typically used to locate objects and boundaries (lines, curves, etc.) in images. In short we can say, image segmentation is the process of assigning a label to every pixel in an image such that pixels with the same label share certain visual characteristics.

As said earlier Region Growing [1] offers several advantages over conventional segmentation techniques. Here the borders of regions are perfectly thin and connected. The algorithm is very stable with respect to noise. Until the parameters are defined correctly the region never contains too much of background. Most importantly, membership in a region can be based on multiple criteria. The image properties such as low gradient or gray level intensity value are also taken into account.

Region Growing [5] is an approach to image segmentation in which neighboring pixels are examined and added to a region class if no edges are detected. This process is iterated for each boundary pixel in the region. If adjacent regions are found, a region-merging algorithm is used on weak edges are dissolved and strong edges are left in tact.

Region Growing offers several advantages over conventional segmentation techniques [6]. Unlike gradient and Laplacian methods, the borders of regions found by region growing are perfectly thin (since we only add pixels to the exterior of our region) and connected. The algorithm is also very stable with respect to noise. Our region will never contain too much of the background, so long as the parameters are defined correctly. Seed Based Region Growing Technique is presented for Breast Cancer Detection and Embedded Expert System[7]. Other techniques that produce connected edges, like boundary tracking [9], are very unstable. Most importantly, membership in a region can be based on multiple criteria. We can take advantage of several image properties, such as low gradient or gray level intensity value, at once. Hybrid method uses both boundary based and region growing method [12]. To resolve the existing work issues, in this work, we implement a local threshold texture image segmentation for natural and aerial images.

\section{Proposed Massive Texture Image Segmentation For Natural And Aerial Images}

The proposed massive texture image segmentation method [MTIS] is applying texture feature-based multitude regional image segmentation on aerial and natural image. A user interaction environment is less in the proposed scheme and assists to the novice computer users. For natural and aerial image segmentation, 2D semiautomatic SRG with texture feature is deployed. After evaluating the features of aerial and natural images with morphological filter and watershed methods, feasible texture features are found.

For aerial and natural image, local threshold selection based segmentation procedure has been developed. A look up table has been maintained to control the homogeneity and statistical values of each pixel in the specified image. Based on the computation of local statistical values, 11 x 11 window size is deployed. This choice is based on the small homogeneous regions, which are produced by the granularity. The window sizes of the natural and aerial images are large enough for the dimension of homogeneity region principle and statistical relationship bound. The selection of parameter of the similarity bound processed depends on the 
granularity or speckle into the images. At first, the seeded growing region algorithm is deployed with the local threshold values. The preliminary growing region reveals the huge number of false consistent region into the image, which was connected with their adjacent region by merging. The constraints for integrating criteria depend on the elevated frequency objects for instance segmentation. This algorithm can be utilized for completely urbanized speckle images with proficient segmentation. The combined regions decrease over segmentation devoid of using further smoothing into the image. The absolute segmentation results display precise consistent regions devoid of implementing texture-based analysis. The diagram 3.1 given below illustrates how the image is segmented provided with the input training images.

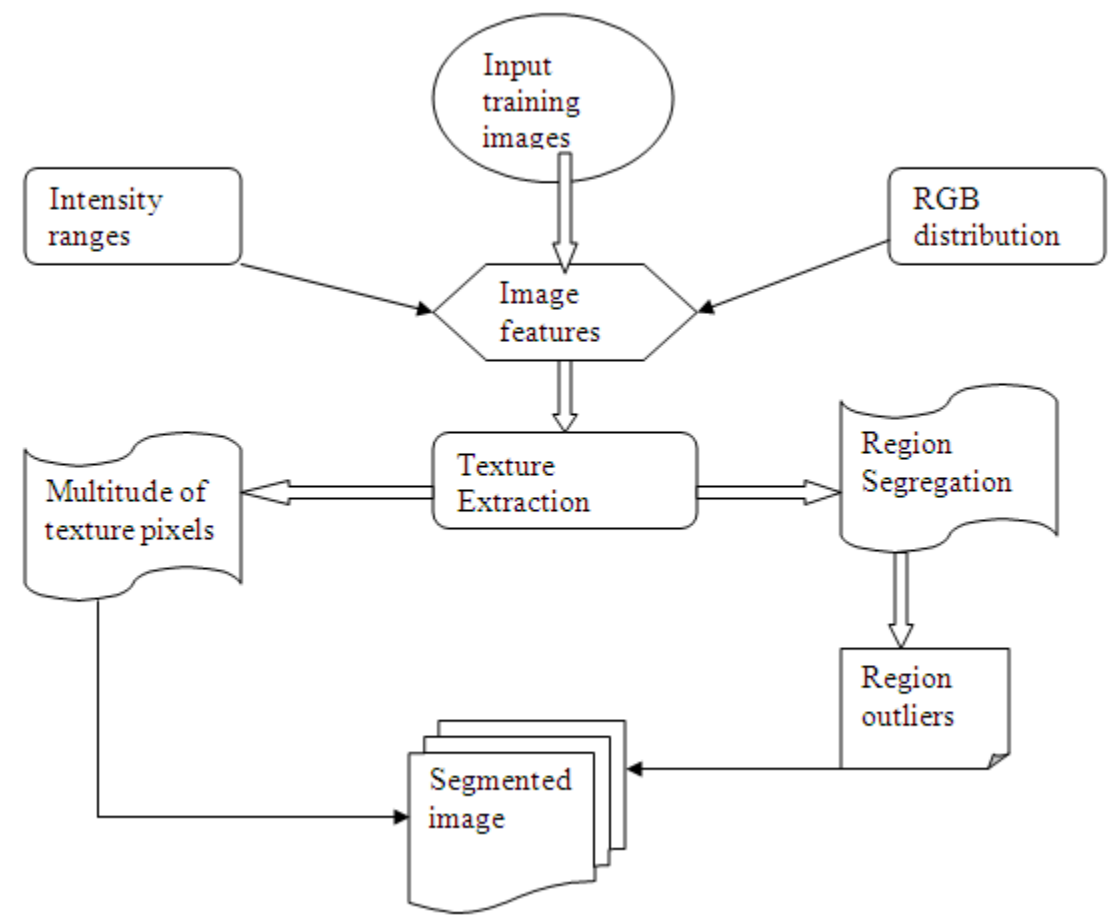

Fig 3.1 Architecture diagram of the proposed MTIS scheme

Fig 3.1 describes the training set with input data from image, intensity ranges and RGB and an alpha value is applied as input to the features extracted from the training images. The RGB image is then converted to image features which are more suitable for determining the borders among the regions. To identify the intensity ranges of determining the popular approaches are

- Threshold techniques,

- Edge-based methods,

- Region-based techniques and

- Connectivity-preserving methods

The proposed multitude region-based method usually works as follows:

Step 1: The input training image is further divided into regions by means of grouping the neighboring pixels that has the similar intensity ranges.

Step 2: The regions that are closest to the pixels are then combined based on some condition such as the RGB value or the alpha distribution value attained.

Step 3: Identify the textures for the region based texture segmentation.

We have also revealed that the texture extraction can also be used for certain tasks such as region segregation and region outliers. Then the extracted textures are applied for the multitude of texture pixels with the multi texture function. The resultant image obtained is the segmented image.

Consider a finite training set of images $S=\left\{\mathrm{s}_{1}, \mathrm{~s}_{2}, \ldots \mathrm{s}_{\mathrm{n}}\right\}$ and can be represented by a set $\mathrm{D}$.

$$
\begin{aligned}
& \sum\left(\frac{1}{2}\left|S_{i}+\alpha D_{i}\right|+\lambda(R G B)_{i}\right) \\
& \text { Where } \mathrm{i}=1,2 \ldots \mathrm{n}
\end{aligned}
$$

Where $\lambda$ signifies the intensity ranges and ói signifies the alpha distribution and (RGB) $)_{i}$ denotes the RGB value for a single image. 
Texture extraction is accomplished in two steps using the set $\mathrm{D}$ and summing it up. It is done by using $\mathrm{S}=\left\{\mathrm{s}_{1}, \mathrm{~s}_{2}, \ldots, \mathrm{s}_{\mathrm{n}}\right\}$. Where $\mathrm{S}_{\mathrm{i}}, \mathrm{i}=1,2 \ldots \mathrm{m}$ are the patches hauled out with size $\mathrm{d} * \mathrm{~d}$ from texture images in training set.

Assume and take the average of all the coefficients for particular texture image values.

$$
\begin{aligned}
& T(S)=\sum_{i=1}^{n}\left(\alpha_{i}\right) \ldots \text { (eqn 2) } \\
& \text { Outlierrate }=(x / n) * 100
\end{aligned}
$$

Where $\mathrm{x}$ signifies the number of outliers obtained and $\mathrm{n}$ signifies the total number of training images. Texture coordinate Cá among two pixels $\mathrm{C}_{0}$ and $\mathrm{C}_{1}$ is specified by

$$
C \alpha=(1+\alpha) C_{0}+\alpha C_{1}
$$

The algorithm (Fig 3.2) below describes the process of the proposed massive regional texture extraction for natural and aerial images.

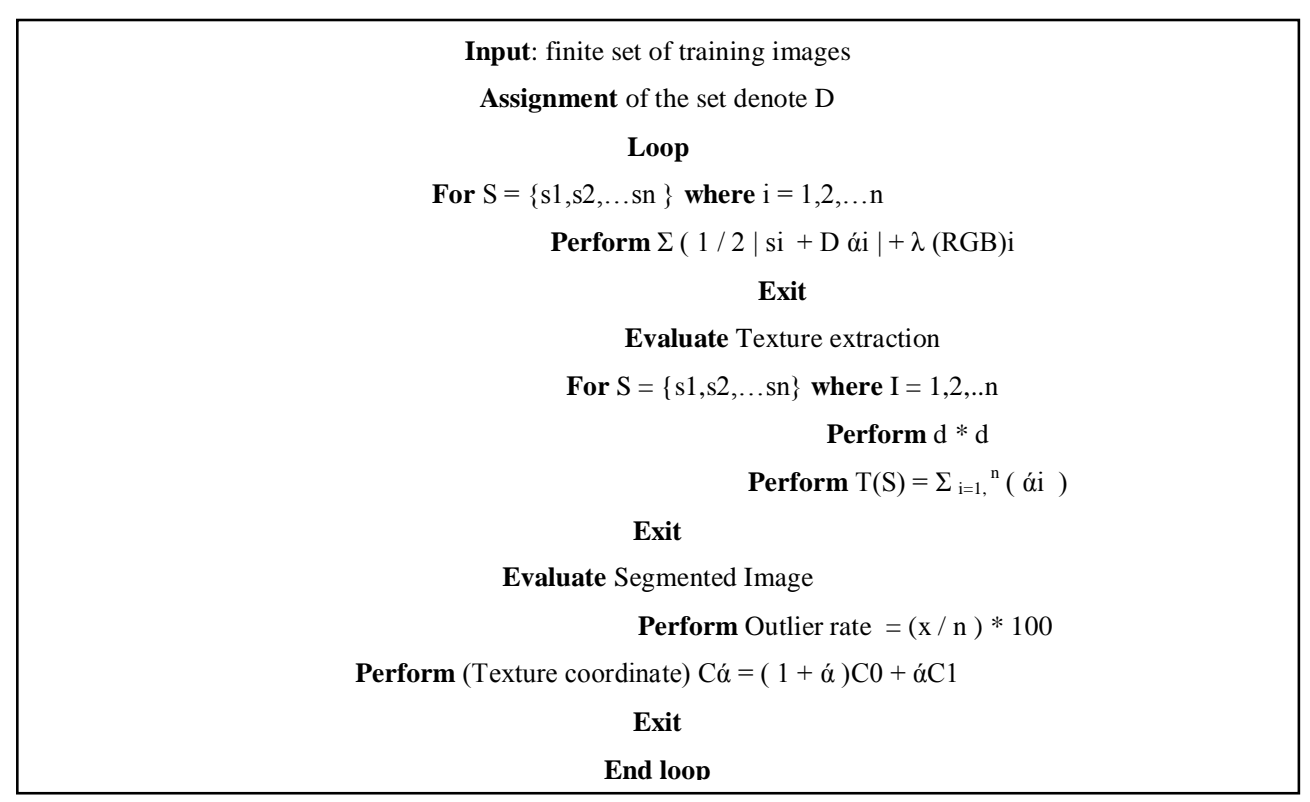

Fig 3.2 Algorithm of the proposed MTIS scheme

In this paper, a local threshold based multitude texture regional seed segmentation is proposed for medical image segmentation. Here, lookup table is maintained for local statistics to use in initial region. Then pixels are combined to assure precise homogeneity criteria. At last it creates a uniform region, and integrating the adjacent regions, which have analogous intensity values.

Algorithm implementation is accomplished by using seeded region growing procedure where each pixel is specified as seed point. The shape and size of the budding regions is done based on look up table entries. The region absorption is completed after the region growing. This contains the elevated frequency objects. The complex regions output will be in the outline of segmented image with superior efficiency than watershed method. It also allows a segmentation of the precise homogeneous regions compared with morphological filter segmentation.

\section{Experimental Results On Multitude Regionaltexture Extraction}

The experimentation conducted on aerial and natural images is to evaluate the performance of proposed local phase and threshold texture extraction for future Natural segmentation [MTIS]. Implementation of the proposed MTIS algorithm is done in MATLAB. In addition to noise removal, the proposed MTIS model also present qualitative results for the texture extraction of the natural image edges. The localization accuracy of natural surface detection technique and assessing the accuracy of measuring relative inner layers of separation is 
a clinically relevant task for which the system uses $2 \mathrm{D}$ imaging. The screen shots of the natural in this proposed MTIS technique is shown in figure given below.

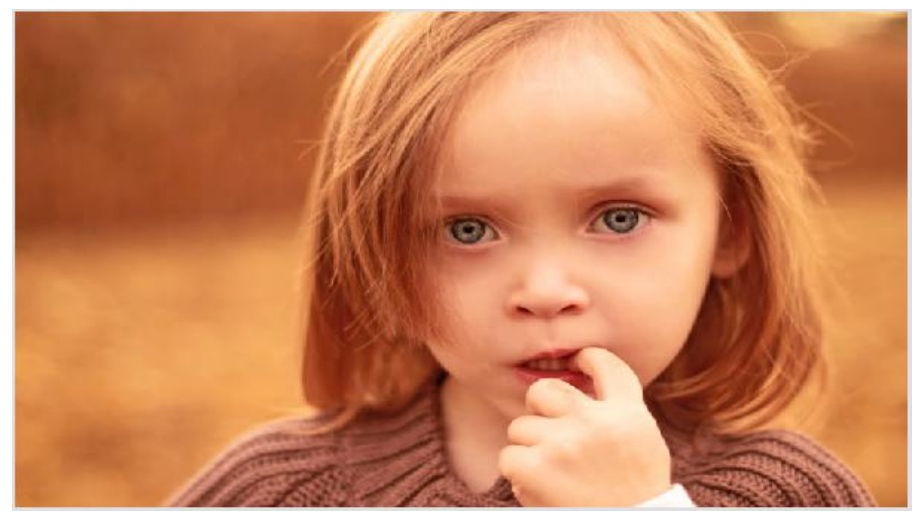

(a) Input Image

\section{x- Direction Image}

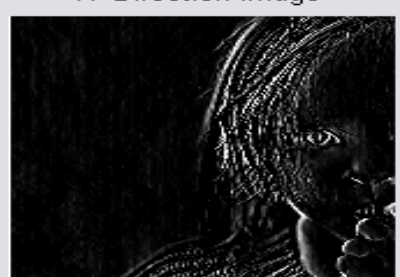

Non-Max Suppress

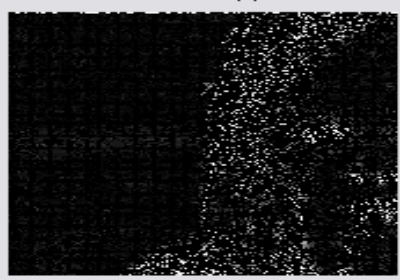

$$
\checkmark \text { - Direction Image }
$$

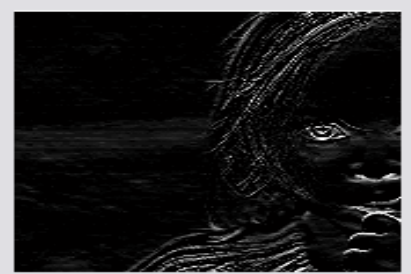

Canny Edge

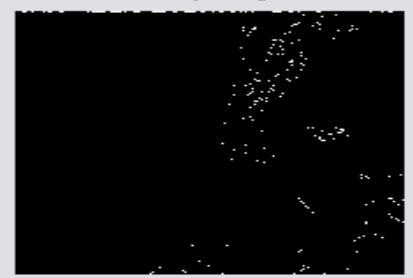

(b)EdgeDetected

Image Texture variance of Gabor filter
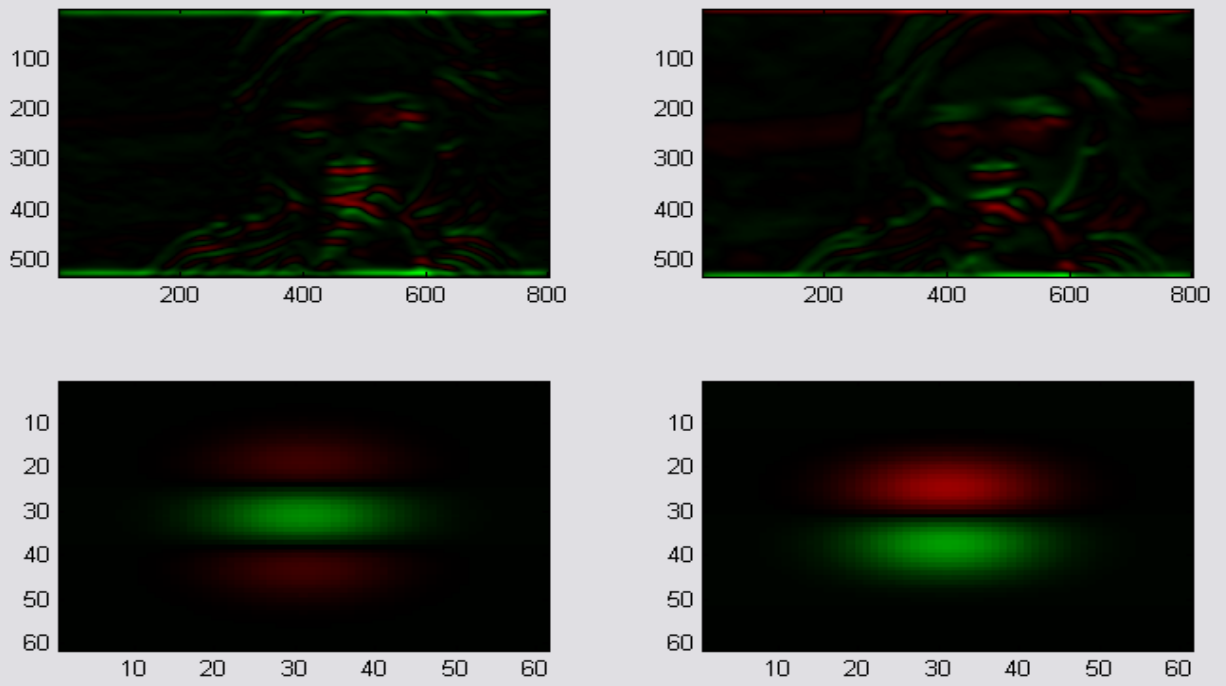

(c) Texture Image 
The datasets used for experimental evaluation are segmented homogeneous region with number of images and number of pixels in segmented region. The experimentation accomplished here provides precise homogeneity criteria and create the consistent region, and integrating the neighboring regions, which contain parallel intensity values.

This can be particularly useful for group work or to beginner computer users. The segmentation of aerial and natural image is deployed as a 2D semi-automatic SRG with texture feature. They are compared with the morphological filter (12\% less homogeneous regions) and watershed methods (15\% less homogeneous pixels) and established to be a possible texture feature in natural and aerial image segmentation.

The performance of the datasets that are compared with the existing system with segmented homogenous region and normal segmented region of threshold technique is given below.

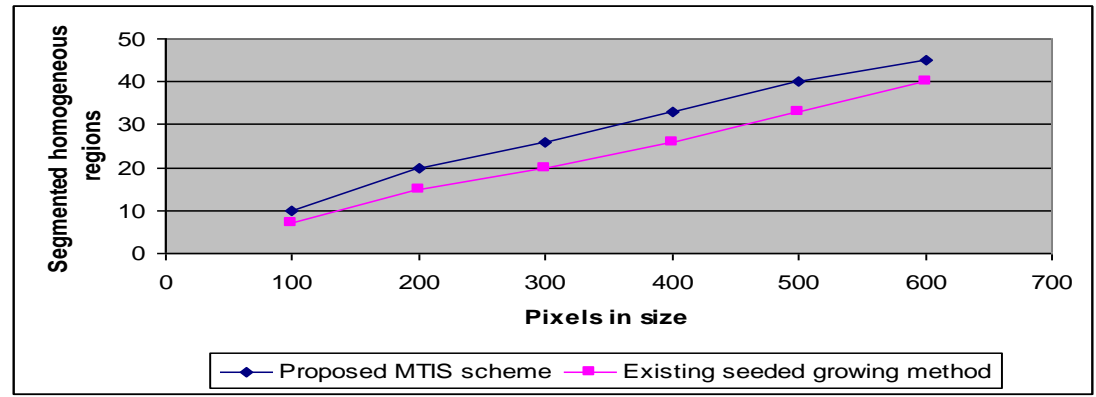

Fig 4.1 Pixels in size vs. segmented homogeneous regions

Fig 4.1 describes the process of segmenting the homogeneous regions of the given aerial and natural images. Based on the pixels size of the image, the segmented homogeneous regions are measured. Compared to the existing seeded growing method, the proposed MTIS scheme increases the homogeneous segmented regions in the range of $40-50 \%$ dramatically.

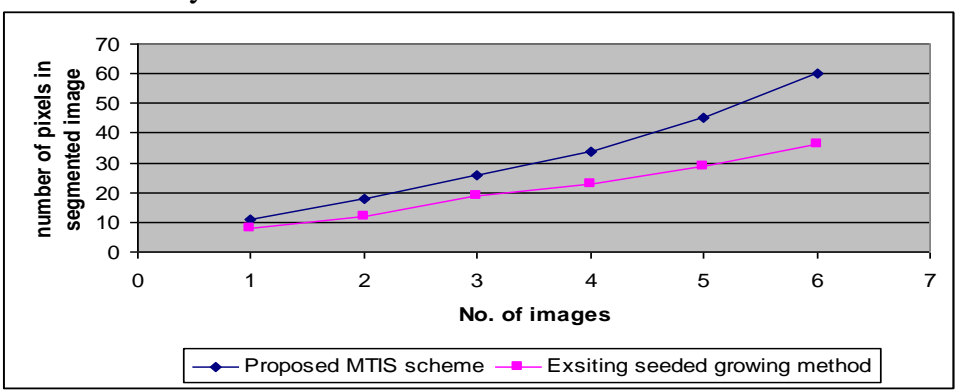

Fig 4.2 No. of images vs. no of pixels in segmented image

Fig 4.2 describes the number of pixels obtained with the segmented regions are measured for the given aerial and natural images. Based on the number of images found, the number of pixels in the segmented homogeneous regions is calculated. Compared to the existing seeded growing method, the proposed MTIS scheme increases the pixels size in the range of $50-60 \%$ dramatically.

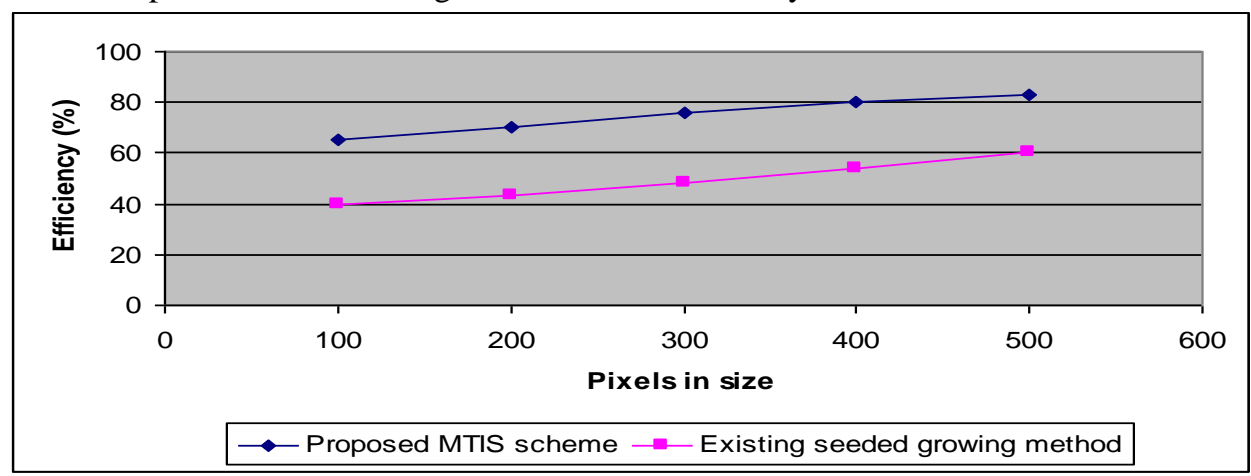

Fig 4.3 Pixels in size vs. efficiency

Fig 4.3 describes the efficiency of the proposed MTIS scheme based on the number of pixels obtained in the input image. The proposed MTIS scheme is efficient since it followed the process of implementing the local threshold values and seeded growing method. Compared to the existing works, the proposed MTIS performed well and the variance is $40-50 \%$ high. 
Finally, it is being observed that the proposed MTIS scheme efficiently done the regional texture extraction methods based on the multitudes of aerial and natural images.

\section{Conclusion}

In this paper, we were successfully implemented massive regional texture extraction methods for aerial and natural images. At first, a local threshold value is initiated to ignore the spatial information and blurred region boundaries. After that the region merging is done to smother the high frequency objects. The region that is merged output will be in the outline of segmented image with higher efficiency than the existing works like watershed method. The proposed MTIS algorithm developed for efficient segmentation to show sharp and fine edges of the segmented portion of the images. Segmented edge detection is accomplished with multitudes of pixels on its divided image portions for pixel variance on any specified image. Multitudes of textural region boundaries and edges are closely related, since there is often a sharp adjustment in intensity at the region boundaries. Experimental results have shown that the proposed MTIS scheme efficiently segment the images by egion outliers and multiture regional extraction schemes. The proposed MTIS scheme is efficient in segmenting the homogeneous regions comapred to the existing seeded region growing model.

\section{References}

[1] T.-Y. Law and P. A. Heng, (2000) "Automated extraction of bronchus from 3-D CT images of lung based on genetic algorithm and 3-D region growing”, Proc. SPIE 3979, Medical Imaging 2000: Image Processing, 906-916.

[2] R. Susomboon, D. S. Raicu, and J. D. Furst, (2006)“Pixel-Based Texture Classification of Tissues in Computed Tomography", CTI Research Symposium, Chicago, April 2006.

[3] J. E. Koss, F. D. Newman, T. K. Johnson, D. L. Kirch, (1999) "Abdominal organ segmentation using texture transform and a Hopfield neural network", IEEE Trans. Medical Imaging, Vol.18, 640-648.

[4] R. Adams, L Bischof, (1994) "Seeded region growing”. IEEE Transaction Pattern Analysis Machine Intelligency 16, 641-647.

[5] N. A. Mat-Isa, M. Y. Mashor \& N. H. Othman, (2005) "Seeded Region Growing Features Extraction Algorithm; Its Potential Use in Improving Screening for Cervical Cancer". International Journal of the Computer, the Internet and Management (ISSN No: 08587027) Vol. 13. No. 1 January-April

[6] Y. Tuduki, K. Murase, M. Izumida, H. Miki, K. Kikuchi, K. Murakami \& J. Ikezoe (2000). "Automated Seeded Region Growing Algorithm for Extraction of Cerebral Blood Vessels from Magnetic Resonance Angiographic Data" Proceedings of The 22nd Annual International Conference of the IEEE Engineering in Medicine and Biology Society 1756-1759

[7] P. A. Venkatachalam, U. K.Ngah, A. F. M. Hani\& A. Y. M. Shakaff, (2002). "Seed Based Region Growing Technique in Breast Cancer Detection and Embedded Expert System". Proceedings of International Conference on Artificial Intelligence in Engineering and Technology 464-469

[8] V. A. Kovalev, F. Kruggel, H.-J Gertz, and D.Y. von Cramon. (2001) "Three-dimensional texture analysis of MRI brain datasets" IEEE Trans. on Medical Imaging, 20(5): 424-433.

[9] S. A. Karkanis, et al., (1999) "Detecting abnormalities in colonoscopic images by texture descriptors and neural networks," Proc. of the Workshop Machine Learning in Med. App., 59-62.

[10] A. Madabhushi, M. Feldman, D. Metaxas, D. Chute, and J. Tomaszewski. (2003) "A novel stochastic combination of 3D texture features for automated segmentation of prostatic adenocarcinoma from high resolution MRI." Medical Image Computing and Computer-Assisted Intervention, volume 2878 of Lecture Notes in 8 J. Wu et al. / J. Biomedical Science and Engineering 2 (2009) 1 8 Computer Science, pp. 581-591. Springer-Verlag

[11] B. W. Whitney, N. J. Backman, J. D. Furst, D. S. Raicu, (2006) "Single click volumetric segmentation of abdominal organs in Computed Tomography images", Proceedings of SPIE Medical Imaging Conference, San Diego, CA, Februar.

[12] J. Wu, S. Poehlman, M. D. Noseworthy, M. Kamath, (2008) Texture Feature based Automated Seeded Region Growing in Abdominal MRI Segmentation, 2008 International Conference on Biomedical Engineering and Informatics, Sanya, China, May 2730 . 